\title{
Independent evolutionary reductions of the phallus in basal birds
}

\author{
Patricia L. R. Brennan, Tim R. Birkhead, Kristof Zyskowski, Jessica van der Waag \\ and Richard O. Prum
}

P.L.R Brennan (correspondence), K. Zyskowski and R. O. Prum, Department of Ecology and Evolutionary Biology, and Peabody Natural History Museum, Yale University, New Haven, CT 06520-8105, USA. E-mail: patricia.brennan@yale.edu. - T. R. Birkhead, Department of Animal and Plant Sciences, University of Sheffield, Western Bank, S10 2TN. Sheffield, UK. - J. van deer Waag, The Center of excellence in Natural Resource Management and the School of Animal Biology, University of Western Australia, Ongerup WA 6336, Australia.

\begin{abstract}
Despite a long history of anatomical studies in birds, the genitalia of most avian species remain undescribed. Birds are the only vertebrate taxon with internal fertilization where an intromittent phallus has been lost in most species. Studying the anatomical transitions of the avian phallus in those species where it is still present, allows us to test evolutionary hypotheses of why the phallus was lost in the ancestor of modern birds. As part of an anatomical survey of the evolution of avian phallus morphology, we have examined some avian species whose genitalia have not been described. Previously, there were only two known events of phallus reduction in birds: one transition from intromittent to non-intromittent in the Galliformes, and a complete loss of phallic structures in the ancestor of Neoaves. Here we report three additional cases of phallus reduction in birds: a transition from intromittent to non-intromittent phallus in Tinamiformes (Crypturellus, Tinamidae), the presence of a non-intromittent phallus in Alectura (Megapodidae), and a complete loss of the phallus in Leipoa (Megapodidae). In addition, we report on the unique morphology of the Crypturellus non-intromittent phallus. These new records of phallus reduction highlight the dynamic nature of phallus evolution in birds. Our findings provide evidence against the hypothesis that the phallus in birds is maintained to insure paternity in taxa with exclusive male parental care, since both groups where we report phallus reduction provide predominately male-only care.
\end{abstract}

Birds are the only vertebrate taxon with internal fertilization where phallic structures have been lost in most species (Briskie and Montgomerie 1997). Only a few groups of birds have retained the ancestral phallus, including the ratites, Tinamiformes, Galliformes and Anseriformes (Gerhardt 1933, Briskie and Montgomerie 1997). Avian phalli are classified in two main types: intromittent phallus protrudens and non-intromittent phallus nonprotrudens (King 1981). The shaft of the intromittent type protrudes from the ventral proctodeal wall of the cloaca, while the non-intromittent type remains attached to it (King 1981), and it is therefore unlikely that it penetrates the female vagina. A recent review of avian phallus evolution suggested a single evolutionary transition between intromittent and non-intromittent phallus within the Galliformes, and a single evolutionary loss in the sister group to Galliformes and Anseriformes (Galloanserae) which is the common ancestor of most living Neoaves (Montgomerie and Briskie 2007).

The anatomy of non-intromittent avian phalli has been well described in the domestic fowl Gallus gallus (Fig. 1a) and turkey Meleagris gallopavo (Phasianidae, Galliformes) (reviewed by King 1981). The non-intromittent phallus in Gallus has four main components: 1) median and lateral phallic bodies (corpus phallicum medianum: c.ph.m. and corpora phallica laterallia:c.ph.l.), 2) lymphatic folds (plycae lymphaticae: l.f.; Fig. 1a), 3) paired peripheral lymphatic sinuses of the paracloacal vascular body (lymphobulbus phalli: l. ph.), which produce the lymph responsible for the engorgement of the phallus during ejaculation, and 4) muscles of the phallus (King 1981, King 1993). The non-intromitent phallus in Meleagris lacks the median phallic body, while the lateral phallic bodies are more developed than in Gallus. The lymphatic folds are also larger and are located on top rather than alongside the lateral phallic bodies as they are in Gallus. During erection the turkey phallus has a double apex, rather than the single apex evident in the chicken, due to the presence of the median phallic body (King 1981).

Despite centuries of work on avian anatomy, avian genitalia are still poorly known, and further work is required to fully describe genital anatomy of many species (Montgomerie and Briskie 2007). In tinamous for example, only the intromittent phallus of the spotted tinamou Nothura maculosa has been fully described (Oliveira and Mahecha 2000). Other tinamou species have an intromittent phallus including the red-legged tinamou Rhynchotus rufescens, white-bellied nothura Nothura boraquira, lesser nothura Nothura minor, grey tinamou Tinamus tao and solitary tinamou Tinamus solitarius (Oliveira et al. 2004). Gerhardt (1933) had previously described the phallus of red-legged tinamou (which he referred to as Tinamus 


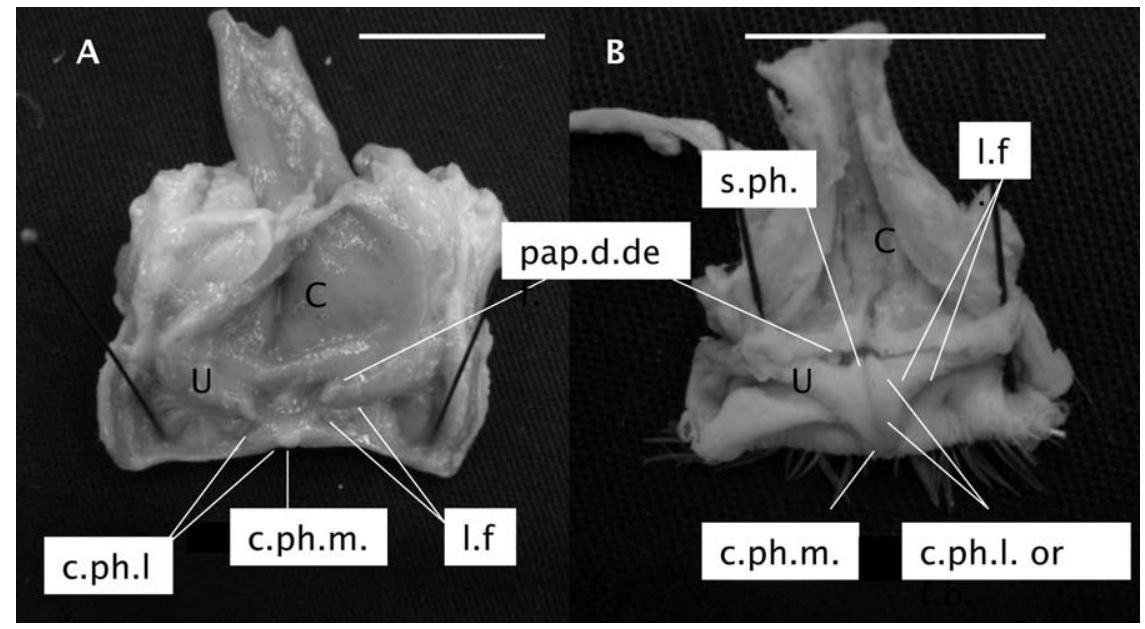

Figure 1. Phallus non-protrudens Dorsal view of dissected cloaca in A. Gallus and B. Crypturellus. The median phallic body (c.ph.m) is white and evident in the chicken, while possibly present at the tip of the tongue-shaped phallus of Crypturellus, where the lateral phallic bodies (c.ph.l.) merge. The c.ph.l. are solid well-developed structures in B, while they are small and inconspicuous in A. There is a clear phallic sulcus (s.ph.) present in B, that is absent in chicken except during erection. The c.ph.l. are rigid in B while soft in A. The papillae of the ductus deferentis (pap.d.def). are well developed in both. $\mathrm{C}=$ Coprodeum, $\mathrm{U}=$ Urodeum.. Scale bars $=2 \mathrm{~cm}$.

rufescens), as being $2 \mathrm{~cm}$ long and resembling that of the kiwi (Apteryx) on a reduced scale (Gerhardt 1933), while King (1981) mistakenly reported that Gerhardt's description was that of the thicket tinamou C. cinnamomeus. Oliveira et al (2004) reported that small-billed tinamou Crypturellus parvirostris, and tataupa tinamou C. tataupa had a non-intromittent phallus, but no anatomical descriptions were provided and findings were not placed in an evolutionary context. In our study of phallus diversity and evolution in birds, we have examined the genitalia of several species and here we describe a novel type of nonintromittent phallus in the genus Crypturellus (Tinamidae), which represents a second example of a transition between intromittent and non-intromittent phalli in birds.

One of the avian families whose phalli are not described are the megapodes (Megapodidae). The presence/absence of the phallus was deemed equivocal by the latest review on avian intromittent organs (Montgomerie and Briskie 2007) because there are no published descriptions of the phallus in this group. The phallus was reported as present in the Australian brush turkey Alectura lathami and the malleefowl Leipoa ocellata, and absent in at least some of the species of the genus Megapodius, based on personal communications published originally by Brom and Dekker (1992). In this study, we describe the phallus anatomy of two megapode species, and contrary to the report by Brom and Dekker (1992), we report the complete absence of phallic structures in the malleefowl, which represents an independent loss of the phallus in birds. We also report a reduction of phallic structures from intromittent to non-intromittent in the Australian brush turkey, and discuss the evolutionary implications of these findings.

\section{Methods}

We examined the macroanatomy of two formalin preserved specimens of undulated tinamou Crypturellus undulatus, collected in Bolivia (YPM136993 and 136994), and spirit specimens of tataupa tinamou (C. tataupa, AMNH, fluid 11470), variegated tinamou (C. variegatus, $A M N H$, fluid 10414), and little tinamou (C. soui AMNH, fluid 8535). We assessed the reproductive status of the males by looking at the size of their testes and the presence of convolutions inside the terminal portion of the Ductus deferentis. All Crypturellus specimens were in peak reproductive condition as evinced by their enlarged testes and well-developed Ducti. We emptied the contents of the terminal portion of one duct into an Eppendorf tube with 200ul of buffered formaline $10 \%$, and examined a $10 \mathrm{ul}$ drop under the microscope $(20 \times)$ to look for sperm cells. Sperm was found in the samples of all Crypturellus males examined.

Within the Tinamidae we also have examined the everted phallus of recently killed red-winged tinamou (YPM136980, 136982-84), ornate tinamou (Nothoprocta ornata: YPM136965 and 136967), Darwin's tinamou (Nothura darwinii YPM 136988-91), white-bellied nothura (Nothura boraquira YPM136872, YPM136874-75, YPM136877,78), and Andean tinamou Nothoprocta pentlandii (TV867,868). All specimens collected were at peak reproductive condition. The phallus of 16 live great tinamous (Tinamus major), and one specimen (YPM136959) from this group were examined in a captive population in Costa Rica during the reproductive season.

In addition, we examined the cloaca of two adult male malleefowl collected during the breeding season (YPM137671 and 137673). Although the testes were enlarged in both specimens, the Ducti were not fully developed (no convolutions were evident) indicating that the males were not in peak reproductive condition. However the avian phallus is present year round despite circannual changes in its size related to breeding seasonality (Hohn 1960, P. Brennan unpubl. data), so we expected to be able to describe the phallus even if was not at peak development. Finally we examined a live specimen of an adult male Australian brush turkey during the breeding season and made observations on the anatomy of the phallus. 
The cloaca and associated muscles were removed and a longitudinal cut made on the dorsal surface of the cloaca to expose the phallus. The length of the phallic bodies was measured to the nearest $0.1 \mathrm{~mm}$. The terminal portion of the intestine was separated from the floor of the cloaca to expose and measure the vascular bodies in $C$. undulatus and C. variegatus. However, this dissection was not done in C. soui or C. tataupa because their small size and ethanol preservation would have caused unacceptable damage to these museum specimens. To minimize the damage on the preserved spirit specimens only the left testis was removed from the body cavity and measured to the nearest $0.1 \mathrm{~mm}$.

\section{Results}

All the Crypturellus species examined have a non-intromittent phallus that protrudes very little from the ventral floor of the cloaca (Fig. 1b). The phallus is a small, smooth and fleshy lamina with a prominent central groove running from its base almost to its distal tip. In the species examined, the phallus measures between $4.0-9.8 \mathrm{~mm}$, but only protrudes from the floor of the cloaca at the distal end by $1.0-1.4 \mathrm{~mm}$. The fleshy lamina is formed by the two 2 lateral phallic bodies (c. ph. l.), that merge at the tip, apparently with the median phallic body $(c . p h . m$.), to form a single apex. The lateral phallic bodies are homologous to the Corpus phalli (c. ph.) that form the shaft of the intromittent phallus. Both sides of the lamina are symmetrical, except in C. variegatus where the right phallic body is longer causing a slight curve to the left seen at the very tip of the phallus.

The ejaculatory groove and sulcus (sulcus phalli: s. ph.) divides the ventral surface of the phallus and is evident in the non-erect phallus. The sulcus is straight, runs directly between the lateral phallic bodies, and ends at the distal end of the phallus where the two sides merge. The lymphatic folds in Crypturellus run alongside the lateral phallic bodies (Fig. 1b). The phallus is rigid, and in $C$. soui and
C. variegatus there is black coloration to the phallus. The left side of the cloaca was dissected further to expose the left paracloacal vascular body. In $C$. undulatus this oval organ $(3.0 \times 1.1 \mathrm{~mm})$ is located at the root of the vas deferens, and was exposed upon separation of the connective tissue between the muscle and the lower portion of the intestine. The lymphatic sinuses are found in the entire area that surrounds the phallic bodies. The left vascular body in $C$. variegatus is found in the same location and it measures $1.5 \times 1.0 \mathrm{~mm}$. The musculature of Crypturellus phallus is similar to that of Gallus and consists of two pairs of muscles: the $m$. retractor phalli cranialis is inserted on the ventrolateral wall of the proctodeum, while the $m$. retractor phalli caudalis, inserts on the ventral aspect of the tip of the phallus (presumed median phallic body), although the latter is very small in Crypturellus.

The phallus of male Crypturellus differs in fundamental ways from the previously described non-intromittent phalli in galliforms. First, the phallic bodies have a firm structural base that is clearly evident upon dissection, whereas in Gallus, the lateral bodies are very small and are best seen during erection when they fill up with lymph. Second, the ejaculatory groove is clearly visible in the non-erect phallus of Crypturellus. In Gallus, the ejaculatory groove is not present in the non-erect phallus and is formed only during ejaculation by engorgement of the lateral phallic bodies. Third, the tip of the phallus is a single apex, similar to Gallus presumably formed by the $c . p h$. m., but different from the double apex found in Meleagris where the c.ph.m. is absent. Fourth, similar to Gallus, the lymphatic folds in Crypturellus run alongside the lateral phallic bodies, whereas in Meleagris they are on top of the $c$. ph. $l$.

The Cryturellus non-intromittent phallus is also very different from the phallus found in all other tinamous we examined, which have a true intromittent organ that protrudes from the cloaca when everted (Fig. 2a, b).

The cloacae of the two malleefowl examined show a complete absence of phallic structures, while the seminal papillae are clearly visible (Fig. 3a). There is a fold on the

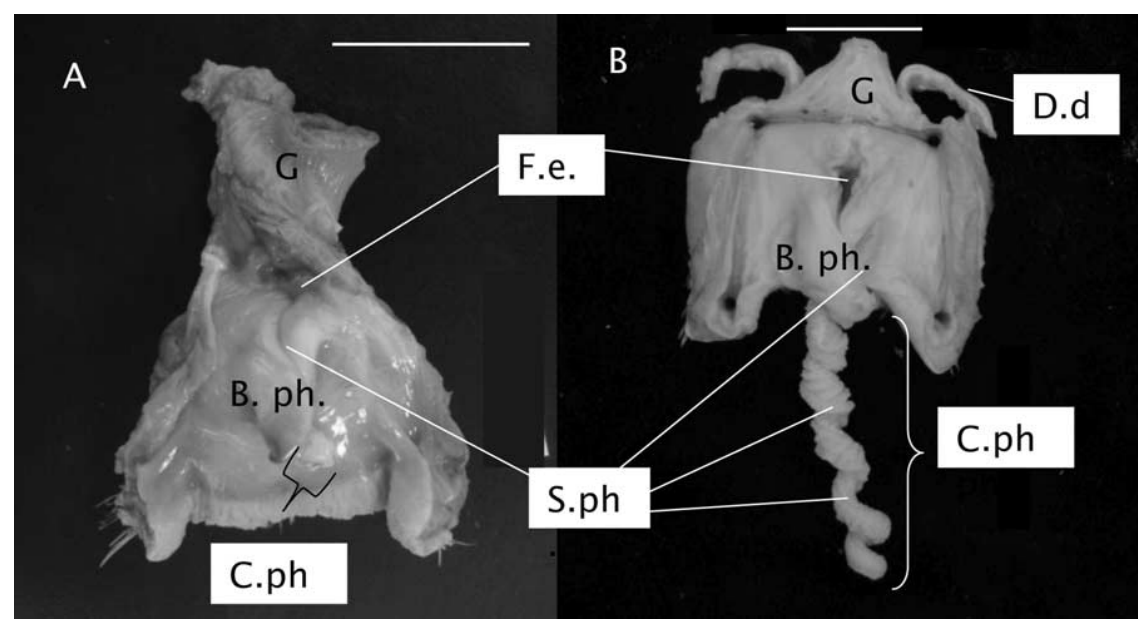

Figure 2. Phallus protrudens in tinamous (Dorsal view) A. Great tinamou (T. major). The phallus has not been everted and the base of the phallus is clearly visible on the ventral surface of the cloaca. The base of the phallus (B. ph.) is rigid and always external. When not everted, the phallus is kept in a phallic pouch. B. Everted phallus of red-legged tinamou ( $R$. rufescens). The body of the phallus (Corpus phalli: $C$. ph.) protrudes from the base and has a shaft made of two phallic bodies (only partially exposed in A). The sulcus phalli (S. ph.) originates at the fossa ejeculatoria (F.e.), where sperm are ejected from the ductus deferentis (D.d.) and runs along the outside of the coiled body of the phallus (C. ph.). $\mathrm{P}=$ Proctodeum, $\mathrm{G}=$ Gut. Scale bars $=2 \mathrm{~cm}$. 


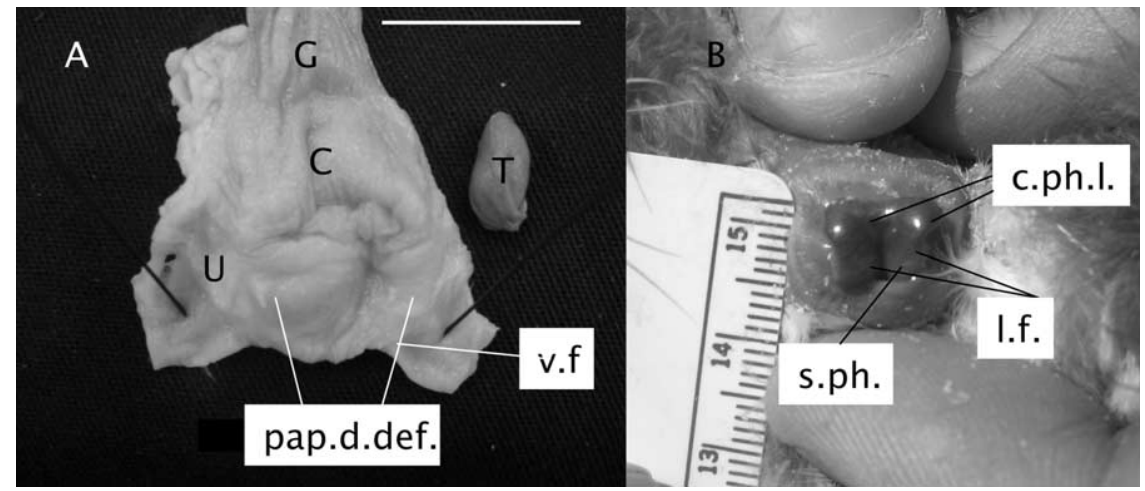

Figure 3. Megapode cloaca. A. Ventral floor of the cloaca in Leipoa (Dorsal view). The phallus is completely absent although there is a ventral fold (v.f.). The papillae of the ductus deferentis (pap.d.def) are clearly seen. B. Non-intromittent everted phallus of Alectura. The phallus lacks a shaft and it resembles that of Meleagris. $\mathrm{C}=$ Coprodeum, $\mathrm{U}=$ Urodeum, $\mathrm{P}=$ Proctodeum, $\mathrm{T}=\mathrm{Testis}, \mathrm{G}=\mathrm{Gut}$. Scale bar $=2 \mathrm{~cm}$.

ventral surface of the cloaca where the phallus is normally present in other species, though the fold varies in prominence between the two specimens examined. No paracloacal vascular bodies were found upon further dissection of the cloaca, while both pairs of phallus muscles are still present.

Finally, the live specimen of Australian brush turkey examined had a non-intromittent phallus (Fig. 3b). A similar conclusion was reached upon examination of a photograph of another brush turkey (provided by D. Jones). There are no phallic bodies that protrude from the ventral floor of the cloaca. The seminal groove splits in two at the distal end of the phallus forming a Y-shaped groove. There does not seem to be a median phallic body as that found in Gallus, and the phallus is superficially similar to that of Meleagris, with a double, rather than a single apex. In addition to the phallus reduction we report in Alectura and Leipoa, the orange-footed scrubfowl Megapodius reinwardt also seem to lack a phallus (D. Jones pers. comm.).

We expanded the avian phylogeny used by Montgomerie and Briskie (2007) to illustrate phallus evolution, by expanding both the tinamou and megapode branches to incorporate our new findings (Fig. 4). Montgomerie and Briskie modified the phylogeny published by Cracraft et al (2004) to present their hypothesis of avian phallus evolution (Montgomerie and Briskie 2007). To Montgomerie and Briskie's hypothesis, we added a simplified version of the most recent tinamou phylogeny resulting from molecular and anatomical characters (Bertelli and Porzecanski 2004). Since all non-Crypturellus tinamous examined by us in this study, and by Oliveira (2004), including 3 species in the sister group to Crypturellus (genus Tinamus), have an intromittent phallus (Fig. 2b), it appears as though the intromittent phallus in tinamous was reduced to nonintromittent only once in the branch leading to all Crypturellus (Fig. 4).

We also added a simplified version of the most recent molecular phylogeny of the megapodes (Birks and Edwards 2002) to the phylogenetic hypothesis in Fig. 4, but the lack of anatomical descriptions of the phallus on most megapodes does not allow us to be certain about its evolution in this group. The phallus could have been independently lost twice within the group, or the loss may have occurred in the ancestor of the family, while it was regained as a nonintromittent phallus later on in brush turkey.

\section{Discussion}

Our discovery of two novel and phylogenetically independent reductions in phallus complexity suggests that phallus evolution has been more dynamic than previously assumed, both qualitatively as we report here, and quantitatively as we reported previously in waterfowl (Brennan et al. 2007).

We have described an anatomically novel type of nonintromittent phallus that represents the second example of an evolutionary reduction of the avian phallus from intromittent to non-intromittent. The differences found between the non-intromittent phalli of Galliformes and Crypturellus may reflect the historical differences in the anatomy of the intromittent phalli of their independent ancestors. Further anatomical work of the Crypturellus phallus is needed, particularly a histological study to describe the microanatomy of the tissues and to understand the functional basis of its distinctly rigid yet reduced phallic bodies.

Even though the malleefowl specimens we examined were not in peak reproductive condition, they were collected during the breeding season and the testes were already starting to enlarge. The phallus should have been detectable under these conditions even if it was not at its peak size. The avian phallus develops in the embryo and it is visible in very young birds (recently hatched), and adults in breeding and non-breeding condition in waterfowl and many tinamous (Tinamidae; P. Brennan unpubl. data). We have no a priori reason to suspect that malleefowl should differ from these other groups in this character. In addition, Dr. D. Jones one of the sources of the personal communications reported by Brom and Dekker (1992), confirmed that Leipoa does not have a phallus, contrary to what was previously reported. The evolutionary changes of the phallus within megapodes cannot be resolved without further anatomical studies of the phallus of other species in this group.

Phallus evolution within the Galliformes is clearly more complex than previously assumed. Based on our current data, the most parsimonious hypothesis of phallus evolution within this group has three evolutionary changes. The last common ancestor between megapodes and other Galliformes 


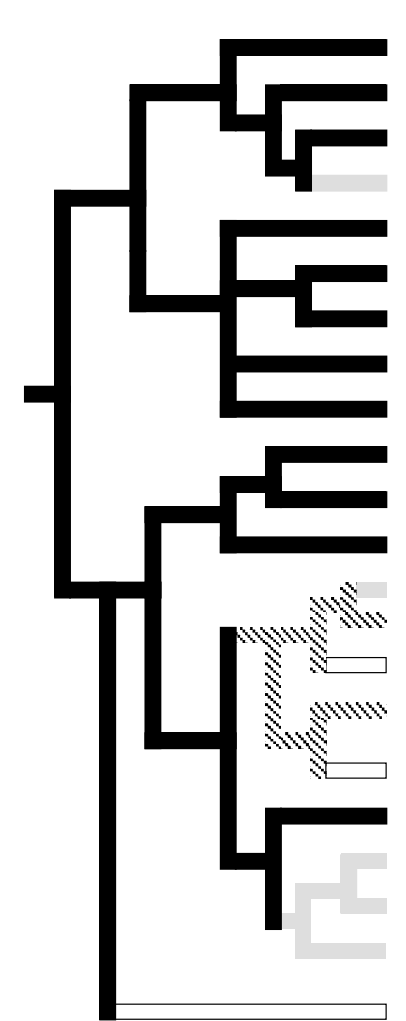

All other tinamids

Nothocercus

Tinamus

Crypturellus

Apterygidae

Casuariidae

Dromaiidae

Struthionidae

Rheidae

Anatidae

Anseranatidae

Anhimidae

Alectura

Aepydodius

Leipoa

Eulipoa

Megapodius

Cracidae

Phasianidae

Odontophoridae

Numididae

All other birds

\section{- Intromittent phallus Non-Intromittent phallus}

\section{$\square$ No phallus \\ … Unknown}

Figure 4. Phylogeny of birds showing the new pattern of avian phallus reduction. New results are highlighted in Bold. The phallus was reduced from intromittent to non-intromittent in the genus Crypturellus and was lost completely in Leipoa and Megapodius, and reduced in Alectura (see text). Phylogeny and phallus evolution modified from Montgomerie and Briskie (2007). Tinamou phylogeny simplified form Bertelli and Porzecanski (2004), Megapode phylogeny simplified from Birks and Edwards (2002).

had an intromittent phallus which was then (1) lost in the ancestor of megapodes, (2) regained as non-intromittent in Alectura, and (3) reduced to non-intromittent in Galliformes other than Cracidae (Fig. 4).

Although an association between promiscuity and phallus presence/absence and morphology has been suggested in birds (Briskie and Montgomerie 1997, Coker et al. 2002, Montgomerie and Briskie 2007), our findings offer mixed support for this relationship. Both Leipoa and M. reinwardt are socially monogamous (Jones 1995), and lack a phallus; whereas the Australian brush turkey is promiscuous and has a non-intromittent phallus. However, several Crypturellus species are promiscuous: females are either polyandrous or both sexes are promiscuous (Davies 2002) and yet the phallus has been reduced in all species examined. Knowledge on the genetic mating system of these poorly known species, may elucidate whether a pattern is evident or not.

The evolutionary significance of the anatomical transitions of the avian phalli remains poorly understood, but our findings offer evidence against one of the few hypotheses to explain phallus maintenance that has been suggested in the literature. The paternity insurance hypothesis states that the phallus has been maintained in certain avian groups because it increases male confidence in paternity presumably by allowing the male to deposit sperm further inside the female cloaca, or to displace sperm from other males (Briskie and Montgomerie 1997). Briskie and Montgomerie (1997) reported an association between mode of parental care and the presence of a phallus (not controlled for phylogeny), where a higher percentage of avian families with exclusive male parental care have a phallus when compared to those where the phallus is absent (Briskie and Montgomerie 1997). Our findings argue against the maintenance of the phallus as a paternity insurance mechanism, since both groups where we report phallus reductions have male incubation and paternal care (Jones et al. 1995, Davies 2002). In addition, although brush turkeys have high levels of cuckolded offspring in their nest mounds (25\%; Birks 1997), and their phallus is nonintromittent, two species that have maintained the intromittent phallus also have high levels of cuckoldry in their nests: ostrich Struthio camelus and emu Dromaius novaengliae males care for up to 68 and $51 \%$ of unrelated chicks (Taylor et al. 2000, Kimwele and Graves 2003), suggesting that an intromittent phallus is not sufficient to insure high levels of paternity, and further casting doubt on the role of the phallus on paternity insurance.

The function of non-intromittent phalli in birds is puzzling, since they clearly are not long enough to penetrate the female vagina to deliver sperm further inside the female oviduct as intromittent phalli do. Non-intromittent phalli could be a selectively neutral intermediate step between a full intromittent phallus and complete phallus loss. However since the underlying erectile machinery are still present, and non-intromittent phalli have diverse morphologies in different taxa, we hypothesize that their function may be to help ensure a more successful copulation, either by providing a better seal with the female cloaca, and/or by stimulating the female during copulation. Functional data on the copulatory mechanics of avian species with nonintromittent phallus is needed to test these hypotheses.

It is clear that phallus evolution in birds is dynamic and complex and that so far we lack a hypothesis that explains the general pattern of phallus presence/absence that we are uncovering. Further anatomical descriptions of morphological changes in the avian phallus will help to understand its evolution.

Acknowledgements - We are grateful to Miguel Angel Aponte from the Noel Kempt Museum in Santa Cruz, Bolivia and Thomas Valqui from Lousiana State University and CORBIDI in Peru for help collecting the tinamou specimens. Darryl Jones kindly provided an image of Alectura phallus for our examination and information on the phallus absence in Megapodius reinwardt. Malleefowl specimens were obtained through the Malleefowl Preservation Group and the Department of Environment and Conservation of Western Australia, collecting permit number SF005707. Ian Gereg and the Livingstone Ripley Waterfowl Conservancy in Lichfield, CT facilitated access to the male Brush turkey for examination. Raul Fournier and ZooAve in Costa Rica helped with tinamou cloacal examination. We are grateful to the curatorial staff at the American Museum of Natural History in New York City, NY, and the Yale Peabody Museum, New Haven, CT who gave us permission to dissect their specimens. Research 
was approved in Bolivia with permit CBF: CITE 032/2006 from the Collección Boliviana de Fauna and it followed all the protocols of animal research IACUC permit \# 2005-10906. Research on captive tinamous in Costa Rica was approved by the MINAE permit number 071-2008. This research was funded through a National Science Foundation postdoctoral grant to PLRB, and work in Bolivia was partially funded by the Yale Peabody Museum at Yale University. We are indebted to Robert Montgomerie and James Briskie for providing helpful comments on this manuscript, and for inspiring our research on avian genitalia.

\section{References}

Bertelli, S. and Porzecanski, A. L. 2004. Tinamou (Tinamidae) systematics: a preliminary combined analysis of morphology and molecules. - Ornitol. Neotrop. 15: 1-7.

Brom, T. G. and Dekker, R. W. 1992. Current studies on megapode phylogeny. - Zool. Verhand. 278: 7-17.

Birks, S. M. 1997. Paternity in the Australian brush-turkey, Alectura lathami, a megapode bird with uniparental male care. - Behav. Ecol. 8: 560-568.

Birks, S. M. and Edwards, S. V. 2002. A phylogeny of the megapodes (Aves: Megapodiidae) based on nuclear and mitochondrial DNA sequences. - Mol. Phyl. Evol. 23: 408421.

Brennan, P. L. R., Prum, R. O., McCracken, K. G., Sorenson, M. D., Wilson, R. E. and Birkhead, T. R. 2007. Coevolution of male and female genital morphology in waterfowl. - PLoS ONE. 2: e418.

Briskie, J. V. and Montgomerie, R. 1997. Sexual selection and the intromittent organ of birds. - J. Avian Biol. 28: 73-86.

Coker, C. R., McKinney, F., Hays, H., Briggs, S. and Cheng, K. 2002. Intromittent organ morphology and testis size in relation to mating system in waterfowl. - Auk 119: 403-413.

Cracraft, J., Barker, F. K., Braun, M., Harshman, J., Dyke, G. J., Feinstein, J., Stanley, S., Cibois, A., Schikler, P., Beresford, P., Garcia-Moreno, J., Sorenson, M. D., Yuri, T. and Mindell, D. P. 2004. Phylogenetic relationships among modern birds (Neornithes): toward an avian tree of life. - In: Cracraft, J. and Donoghue, M. J. (eds). Assembling the tree of life. Oxford University Press. New York, pp. 468-489.

Davies, S. J. J. F. 2002 Ratites and tinamous. Tinamidae, Rheidae, Dromaiidae, Casuariidae, Apterygidae, Struthionidae. Vol 9. - Oxford University Press, Oxford.

Gerhardt, U. 1933. Kloake und Begattungsorgane. - In: Bolk L., Goppert E., Kallius E. and Lubosch W. (eds) Hanbuch der vergleichenden anatomie der wirbeltiere. Vol 6. Urban and Schwarzenberg, Berlin, pp. 267-350.

Hohn, E. O. 1960. Seasonal changes in the mallard's penis and their hormonal control. - Proc. Zool. Soc. 134: 547-555.

Jones, D. N., Dekker, R.W. and Roselaar, C. S. 1995. The megapodes: megapodiidae. - Oxford University Press, New York.

Kimwele, C. N. and Graves, J. A. 2003. A molecular genetic analysis of the communal nesting of the ostrich (Struthio camelus). - Mol. Ecol. 12: 229-236.

King, A. S. 1981. Phallus. - In: King A. S., McLelland J. (eds). Form and function in birds. Vol 2. Academic Press, New York, pp 107-148.

King, A. S. 1993. Apparatus urogenitalis. - In: Baumel, J. J. (ed.). Handbook of avian anatomy. Nuttal Ornithological Club, Cambridge, pp. 329-397.

Montgomerie, R. and Briskie, J. V. 2007. Anatomy and evolution of copulatory structures. - In: Jamieson, B. G. M. (ed.). Reproductive biology and phylogeny of birds. Part A: Phylogeny, morphology, hormones and fertilization Vol 1. Science Publishers, Enfield, pp. 115-148.

Oliveira, C. A. and Mahecha, G. A. 2000. Morphology of the copulatory apparatus of the spotted tinamou Nothura maculosa (Aves Tinamiformes). - Annal Anat. 182: 161-169.

Oliveira, C. A., Silva, R. M., Santos, M. M. and Mahecha, G. A. 2004. Location of the ureteral openings in the cloacas of tinamous, some ratite birds, and crocodilians: A primitive character. - J. Morphol. 260: 234-246.

Taylor, E., Blache, D., Groth, D., Wetherall, J. D. and Martin, G. B. 2000. Genetic evidence for mixed parentage in the nests of emu (Dromaius novaehollandiae). - Behav. Ecol. Sociobiol. 47: 359-364. 